H. Mahajan - C. M. Kolka - J. M. B. Newman •

S. Rattigan - S. M. Richards - M. G. Clark

\title{
Vascular and metabolic effects of methacholine in relation to insulin action in muscle
}

Received: 28 August 2005 / Accepted: 18 October 2005 / Published online: 1 February 2006

C) Springer-Verlag 2006

\begin{abstract}
Aims/hypothesis: Methacholine (MC) is a nitric oxide vasodilator, but unlike other vasodilators, it potentiates insulin-mediated glucose uptake by muscle. The present study aimed to resolve whether this action was the result of a vascular effect of $\mathrm{MC}$ leading to increased muscle perfusion or a direct effect of MC on the myocytes. We hypothesise that vascular-mediated insulin-stimulated glucose uptake responses to $\mathrm{MC}$ occur at lower doses than direct myocyte MC-mediated increases in glucose uptake. Methods: The vascular and metabolic effects of this vasodilator were examined in rats in vivo using a novel local infusion technique, and in the pump-perfused rat hindlimb under conditions of constant flow. Results: Local infusion of low-dose MC $(0.3 \mu \mathrm{mol} / \mathrm{l})$ into the epigastric artery of one leg (test) in vivo markedly increased femoral blood flow and decreased vascular resistance, without effects in the contra-lateral leg. Capillary recruitment, but not glucose uptake, was increased in the test leg. All increases caused by MC were confined to the test leg and blocked by local infusion into the test leg of $N^{\omega}$-nitro-L-arginine methyl ester (L-NAME), but not by infusion of $N^{\omega}$-nitro-D-arginine methyl ester (D-NAME). In the constant-flow pumpperfused rat hindlimb, infusion of $0.6 \mu \mathrm{mol} / 1 \mathrm{MC}$ vasodilated the pre-constriction effected by $70 \mathrm{nmol} / \mathrm{l}$ noradrenaline or $300 \mathrm{nmol} / 1$ serotonin, and this was blocked by $10 \mu \mathrm{mol} / 1$ L-NAME. 2-Deoxyglucose in muscle was increased by $30 \mu \mathrm{mol} / 1 \mathrm{MC}(p<0.05)$, but was unaffected by $3 \mu \mathrm{mol} / 1 \mathrm{MC}$. All increases in 2-deoxyglucose uptake by $30 \mu \mathrm{mol} / 1 \mathrm{MC}$ were blocked by $10 \mu \mathrm{mol} / 1 \mathrm{~L}-\mathrm{NAME}$. Conclusions/interpretation: $\mathrm{MC}$ has dose-dependent effects both on the vasculature and on muscle metabolism. At low dose $(0.3-3 \mu \mathrm{mol} / \mathrm{l}), \mathrm{MC}$ is a potent vasodilator in muscle, both in vivo and in vitro, without metabolic effects;
\end{abstract}

H. Mahajan · C. M. Kolka · J. M. B. Newman · S. Rattigan ·

S. M. Richards $\cdot$ M. G. Clark $(\bowtie)$

Biochemistry, Private Bag 58,

University of Tasmania,

7001 Hobart, TAS, Australia

e-mail: Michael.Clark@utas.edu.au

Tel.: +61-3-62262672

Fax: +61-3-62262703 at higher doses $(\geq 30 \mu \mathrm{mol} / \mathrm{l}) \mathrm{MC}$ has a direct metabolic effect leading to increased glucose uptake. Both the vascular and metabolic effects are sensitive to L-NAME. The lowdose enhancement of insulin action in vivo by MC, which has been reported previously, thus seems to be attributable to vascular effects.

Keywords Capillary recruitment - Glucose uptake · In vivo effects - Perfused muscle effects ·

Total blood flow $\cdot$ Vasodilatation

Abbreviations BP: mean arterial blood pressure . 2DG: 2-deoxyglucose - FBF: femoral arterial blood flow . 5-HT: serotonin - MC: methacholine - 1-MX:

1-methylxanthine $\cdot \mathrm{NA}$ : noradrenaline $\cdot \mathrm{D}-\mathrm{NAME}$ :

$N^{\omega}$-nitro-D-arginine methyl ester · L-NAME:

$N^{\omega}$-nitro-L-arginine methyl ester $\cdot$ L-NMMA:

$\mathrm{N}$-monomethyl-L-arginine $\cdot \mathrm{NO}$ : nitric oxide

\section{Introduction}

Nitric oxide (NO)-producing vasodilators have the potential to exert both vascular-mediated muscle glucose uptake [1-3] and direct myocyte metabolic actions leading to increased glucose uptake in muscle [4]. By lowering vascular resistance and increasing bulk flow, glucose uptake may be enhanced by the increased substrate and hormone delivery. Alternatively, NO production in the myocytes may increase glucose uptake independently of vascular effects, as has been demonstrated with isolated incubated animal muscle systems $[4,5]$.

A number of vasodilators increase limb blood flow in human subjects in vivo and these include adenosine [6], bradykinin [7], sodium nitroprusside [8], low doses of IGF-1 [9] and adrenaline [10]. Nevertheless, none of these has increased glucose uptake or overcome insulin resistance. In contrast to these vasodilators, methacholine (MC), an endothelium-dependent vasodilator, enhances insulinmediated increases in total skeletal muscle blood flow and glucose uptake during a hyperinsulinaemic-euglycaemic 
clamp in human subjects [11]. These effects of $\mathrm{MC}$ were blocked by the NO synthase inhibitor $N$-monomethylL-arginine (L-NMMA) [11]. Because the data indicated a significant deviation from the Renkin equation for a fixed capillary surface area [12], the authors concluded that capillary recruitment was responsible [11]. It was also reported [3] that MC, but not nitroprusside, increased both forearm blood flow and glucose uptake in hypertensive patients. Recently, we have reported that local MC, but not local bradykinin, potentiated insulin-mediated capillary recruitment and glucose uptake in rat muscle in vivo [13]. However, it is not clear that the effect of MC to enhance insulin-mediated glucose uptake by muscle in vivo can be entirely attributed to the enhanced capillary recruitment, particularly since NO donors can increase muscle glucose uptake independently of vascular effects $[4,5]$.

Thus, in the present study we assessed the relationship between the vascular and metabolic effects of MC in rats by comparing in vivo actions with those in the in vitro constant-flow perfused rat hindlimb. The effects of MC that lead to vasodilatation of the pre-constricted pump-perfused rat hindlimb were separated from the actions of MC that influence glucose uptake in the fully dilated hindlimb, where vasodilatory influences on metabolism are minimal.

\section{Materials and methods}

Animals

All procedures adopted and experiments undertaken were approved by the University of Tasmania Animal Ethics Committee. Male hooded Wistar rats weighing $290 \pm 3 \mathrm{~g}$ for in vivo studies, and $190 \pm 3 \mathrm{~g}$ for perfused hindlimb studies, were raised on a commercial diet (Pivot, Launceston, Australia) containing $21.4 \%$ protein, $4.6 \%$ lipid, $68 \%$ carbohydrate and $6 \%$ crude fibre, with added vitamins and minerals, together with water ad libitum. Until the day of surgery, rats were housed at a constant temperature of $21 \pm 1{ }^{\circ} \mathrm{C}$ with a 12 -h light-dark cycle. Food and water were freely available until animals were administered anaesthetic.

\section{Experimental systems}

Two experimental systems were used (Fig. 1). The first system, conducted in vivo, assessed the effect of a local low dose of MC, with and without NO synthase inhibitor, on vascular responses and glucose uptake in one hindlimb, using the contra-lateral hindlimb as control. The low dose of MC used in vivo was identical to that found in previous studies [13] to potentiate insulin action on muscle glucose uptake. The second system of experiments used the surgically isolated pump-perfused hindlimb. Allowing much higher doses of MC than could be confined to one leg in vivo, it enabled assessment of differential dose effects on vascular response and glucose uptake. Fifty animals were used: $n=5$ and $n=6$ per group in vivo and for hindlimb perfusion respectively.

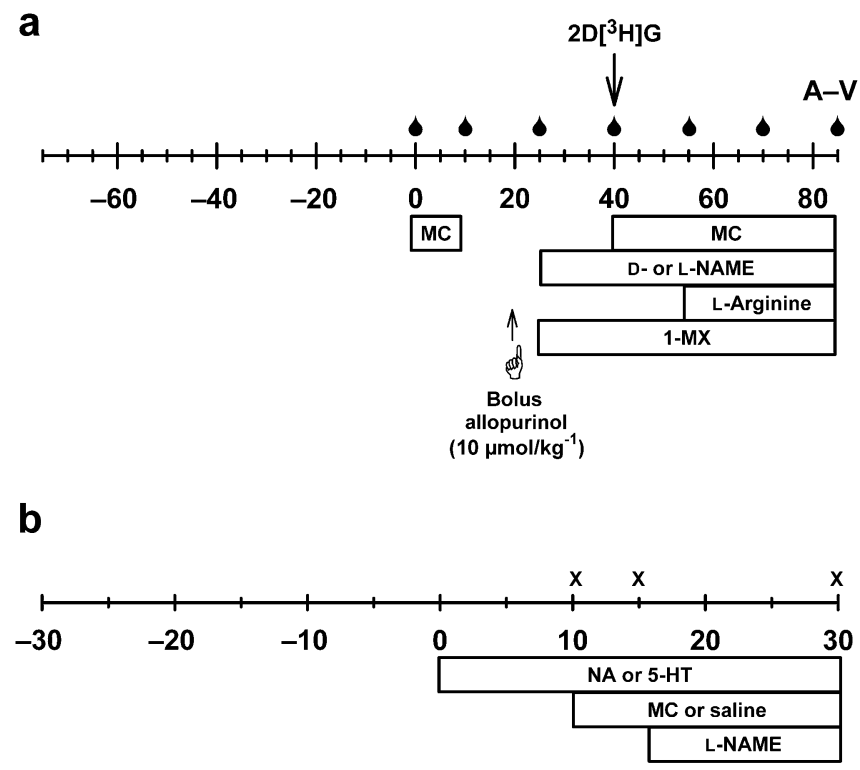

Fig. 1 Experimental protocols for in vivo (a, protocol 1) and hindlimb perfusion studies (b, protocol 2). For in vivo studies (a) surgery and preparation were conducted as described in the text. A 10 -min infusion of MC into the test leg was conducted at $t=0$. An increase in FBF confirmed correct placement of the epigastric cannula for local infusions. The FBF of the test leg was allowed to return to baseline values. Studies on four groups of five animals each were then conducted involving infusion of the following into the epigastric artery of the test leg: D-NAME+MC, L-NAME+MC, $\mathrm{L}-\mathrm{NAME}+\mathrm{MC}+\mathrm{L}$-arginine, and $\mathrm{L}-\mathrm{NAME}$ alone. Infusions (horizontal bars) were adjusted in relation to the FBF of the test leg to attain $0.3 \mu \mathrm{mol} / 1 \mathrm{MC}, 10 \mu \mathrm{mol} / 1 \mathrm{~L}-\mathrm{NAME}, 10 \mu \mathrm{mol} / 1 \mathrm{D}-\mathrm{NAME}$ and $200 \mu \mathrm{mol} / 1 \mathrm{~L}$-arginine in that leg. Systemic infusion of 1-MX $\left(0.5 \mathrm{mg} \mathrm{min}^{-1} \mathrm{~kg}^{-1}\right.$ for $\left.60 \mathrm{~min}\right)$ was preceded by a bolus systemic injection of allopurinol $(10 \mu \mathrm{mol} / \mathrm{kg})$ at $20 \mathrm{~min}$. A bolus systemic injection of $2 \mathrm{D}\left[{ }^{3} \mathrm{H}\right] \mathrm{G}$ was made at $40 \mathrm{~min}$ (arrow). Arterial blood samples were taken for glucose analyses as indicated by the drops. Other arterial samples were taken to monitor the removal of $2 \mathrm{D}\left[{ }^{3} \mathrm{H}\right] \mathrm{G}$ as indicated in the text. The carotid artery and femoral vein of each leg (test and control) were sampled at the completion of the experiment (arterio-venous, A-V). For the hindlimb perfusion studies (b) surgery was conducted as described in the text, and the preparation allowed to equilibrate for $30 \mathrm{~min}$ at constant flow. Infusions are indicated by the horizontal bars. Vasoconstrictors were infused for $10 \mathrm{~min}$ prior to infusion of vehicle or MC. L-NAME was infused $5 \mathrm{~min}$ after MC as indicated. X, time points at which changes in perfusion pressure were analysed

\section{Surgery for experiments in vivo}

Details for positioning cannulae and flow probes in anaesthetised rats were as described previously [13]. Provision was made for the following: maintenance of anaesthesia by constant trickle infusion of Nembutal; measurement of mean arterial blood pressure (BP), heart rate and femoral arterial blood flow (FBF) in each leg; infusion of $\mathrm{MC}$ and/ or NO synthase inhibitors into one (test) leg. Once the surgery was completed, a 45-60-min equilibration period was allowed so that FBF and BP could become stable and constant. Rats were then subjected to protocol 1 (Fig. 1), where they were infused locally (epigastric artery) into the test leg with $\mathrm{MC}$ for the last $45 \mathrm{~min}$. The dose of MC that would produce maximal increase in FBF without effects on 
arterial BP or heart rate was the same as used previously [13]. As shown in Fig. 1, an early 10-min infusion was conducted at $t=0$ to ascertain that the cannulation of the epigastric artery was successful. This is shown on all time course traces. In some experiments local infusion of L-NAME or D-NAME into the test leg was commenced 15 min before MC. In some involving L-NAME infusion, L-arginine was infused for the last $30 \mathrm{~min}$ (Fig. 1, protocol 1).

\section{Experiments in vivo: blood samples}

Arterial samples were taken at the times indicated (Fig. 1, protocol 1) for blood glucose measurements and at -5 and $85 \mathrm{~min}$ for insulin measurements. The femoral vein of each leg was used for venous sampling, using a $29 \mathrm{G}$ insulin syringe (Becton Dickinson, Parsippany, NJ, USA); duplicate samples $(200 \mu \mathrm{l})$ were taken only on completion of the experiment to prevent alteration of the blood flow from the hindlimb due to sampling, and to minimise the effects of blood loss. The blood samples were placed on ice, immediately centrifuged, and the plasma stored at $-20^{\circ} \mathrm{C}$ until assayed. The total blood volume withdrawn from the animals before the final arterial and venous samples did not exceed $1.5 \mathrm{ml}$ and was easily compensated for by the volume of fluid infused over the duration of the experiment.

Experiments in vivo: capillary recruitment

Capillary recruitment was determined by measuring the metabolism of systemically infused 1-methylxanthine (1-MX) as described in detail previously [13]. Plasma $(20 \mu \mathrm{l})$ from arterial and leg venous blood samples taken at the end of the experiment was mixed with $80 \mu$ perchloric acid $(2 \mathrm{~mol} / \mathrm{l})$ and centrifuged for $10 \mathrm{~min}$. The supernatant fraction was used to determine 1-MX concentrations by reverse-phase HPLC, as previously described [10]. Capillary recruitment, expressed as 1-MX metabolism, was calculated from arterio-venous plasma 1-MX difference and multiplied by FBF (corrected for the volume accessible to $1-\mathrm{MX}, 0.871$, determined from plasma concentrations obtained after additions of standard 1-MX to whole rat blood) and expressed as $\mathrm{nmol} / \mathrm{min}$.

Experiments in vivo: muscle glucose uptake

At $45 \mathrm{~min}$ before the completion of each experiment (Fig. 1, protocol 1), a 1.85-MBq bolus of 2-deoxy-D-[2,6- $\left.{ }^{3} \mathrm{H}\right]$ glucose $\left(2 \mathrm{D}\left[{ }^{3} \mathrm{H}\right] \mathrm{G}\right.$; specific activity $1.92 \mathrm{TBq} / \mathrm{mmol}$; Amersham Life Science, Castle Hill, NSW, Australia) in saline $(154 \mathrm{mmol} / \mathrm{l} \mathrm{NaCl})$ was administered systemically. Arterial plasma samples $(25 \mu \mathrm{l})$ were collected at 5, 10, 15, 30 and $45 \mathrm{~min}$ after the $2 \mathrm{D}\left[{ }^{3} \mathrm{H}\right] \mathrm{G}$ bolus to determine plasma clearance of the radioactivity. At the conclusion of the experiment, the soleus, plantaris, gastrocnemius white, gastrocnemius red, extensor digitorum longus and tibialis muscles were removed from the test and control contra- lateral legs, clamp frozen in liquid nitrogen and stored at $-20^{\circ} \mathrm{C}$. Individual frozen muscles were ground under liquid nitrogen and $100 \mathrm{mg}$ muscle tissue was homogenised with $1.5 \mathrm{ml}$ water using an Ultra Turrax (IKA, Wilmington, NC, USA). Free and phosphorylated $2 \mathrm{D}\left[{ }^{3} \mathrm{H}\right] \mathrm{G}$ were separated by ion exchange chromatography using an anion exchange resin (AG1-X8) [14, 15]. Biodegradable Counting Scintillant-BCA (Amersham Life Science) was added to each radioactive sample and radioactivity determined using a scintillation counter (LS3801, Beckman Instruments, Fullerton, CA, USA). From this measurement and a knowledge of plasma glucose and the time course of plasma radioactive 2DG disappearance, muscle 2DG uptake, which reflects glucose uptake into the muscle, was calculated as previously described by others $[14,15]$.

Experiments in vivo: other assays

A glucose analyser (2300 Stat plus; Yellow Springs Instruments, Yellow Springs, OH, USA) was used to determine whole blood glucose (by the glucose oxidase method) during each experiment. A blood sample of $25 \mu \mathrm{l}$ was required for each determination. Insulin assays were conducted on arterial plasma samples taken at $-5 \mathrm{~min}$ and at $85 \mathrm{~min}$ using an ELISA method (Mercodia, Uppsala, Sweden).

\section{Experiments in vivo: expression of results}

All data are expressed as means \pm SE. Means for FBF, heart rate and BP were calculated from 5-s subsamples of the data, representing approximately 500 flow and pressure measurements every $15 \mathrm{~min}$. Vascular resistance in the hindleg was calculated as mean BP in $\mathrm{mmHg}$ divided by $\mathrm{FBF}$ in $\mathrm{ml} / \mathrm{min}$ and expressed as resistance units.

Perfused rat hindlimb studies: surgery

Hindlimb surgery was performed as described by others [16] with additional details by us as outlined elsewhere [17]. In brief, the rats were anaesthetised with Nembutal (5-6 mg/100-g body weight, i.p.) and allowed to breathe room air freely. The tail was ligated and a small portion of the skin over the thigh region removed so that the superficial epigastric vessels could be ligated. Vessels supplying the testes were ligated and the testes removed. Ligatures were also positioned so that the bladder and seminal vesicles, colon, large intestine and duodenum could be removed. Care was taken to tie off the superior and inferior mesenteric arteries. Ligatures were placed around the iliolumbar vessels, renal vessels and the common iliac artery supplying the contra-lateral hindlimb, as only one leg was perfused. The descending aorta and vena cava were cannulated immediately above the bifurcation, the in-flow perfusion line connected to the arterial cannula and the pump activated. The limb and venous outflow line were allowed to clear of blood. The time taken for the 
cannulation of the vena cava and the aorta did not exceed 2 min. After the hindlimb was connected to the perfusion network two further ligatures were positioned and the animal was killed with an overdose of pentobarbital sodium (intracardiac). One ligature was positioned around the tarsus of the perfused leg and another around the abdomen at approximately the level of the L3 vertebrate. Both ligatures were placed to ensure that only the leg was perfused. The entire operation was completed within $20 \mathrm{~min}$ and the animal was kept alive under anaesthesia. If the animal died during the operation, the preparation was discarded.

Perfused rat hindlimb studies: perfusions

Perfusions were conducted using a constant flow of $8 \mathrm{ml} / \mathrm{min}$ with Krebs-Henseleit bicarbonate buffer containing $2.5 \mathrm{mmol} / 1 \mathrm{CaCl}_{2}, 8.3 \mathrm{mmol} / \mathrm{l}$ glucose and $4 \%(\mathrm{w} / \mathrm{v})$ BSA. The perfusate was gassed with $95 \% \mathrm{O}_{2}-5 \% \mathrm{CO}_{2}$ in a silastic tube oxygenator and the temperature was maintained at $32^{\circ} \mathrm{C}$ using a heat-exchanger coil. These conditions have been used previously to examine the effects of vasoactive substances on hindlimb metabolism ([18] and references therein). Perfusion pressure was constantly monitored from an in-line pressure transducer located in the arterial line.

Perfused rat hindlimb studies: vasodilatation of pre-constricted hindlimb

After an equilibration period of $30 \mathrm{~min}$, noradrenaline (NA) or serotonin (5-HT) infusions were commenced to maintain a constant vasoconstriction throughout the experiment (Fig. 1, protocol 2). The final concentrations in the perfusate of NA and 5-HT were 70 and $300 \mathrm{nmol} / \mathrm{l}$, respectively. Ten minutes after the commencement of the above infusions, the $\mathrm{MC}$ infusion $(0.6 \mu \mathrm{mol} / 1$, final $)$ was initiated and $5 \mathrm{~min}$ later L-NAME infusion $(10 \mu \mathrm{mol} / \mathrm{l}$, final) was commenced and continued for 15 min while maintaining the infusion of MC (Fig. 1, protocol 2). Pressure was monitored throughout. A separate series of experiments involved only $70 \mathrm{nmol} / 1 \mathrm{NA}$ and $10 \mu \mathrm{mol} / 1$ L-NAME or $300 \mathrm{nmol} / 15$-HT and $10 \mu \mathrm{mol} / \mathrm{l} \mathrm{L}-\mathrm{NAME}$. The L-NAME infusion was commenced $15 \mathrm{~min}$ after the commencement of NA or 5-HT infusions and was maintained for 15 min (Fig. 1, Protocol 2).

Perfused rat hindlimb studies: 2-DG uptake by lower leg muscles of the fully dilated hindlimb

After equilibration (30 min), a graded infusion of $\mathrm{MC}$ was commenced. The initial concentration of MC was $0.3 \mu \mathrm{mol} / 1$ and this was increased to 3,15 and $30 \mu \mathrm{mol} / 1$ at intervals of $10 \mathrm{~min}$. Controls involved infusion of saline $(154 \mathrm{mmol} / \mathrm{l}$ $\mathrm{NaCl})$ or L-NAME alone. $2 \mathrm{D}\left[{ }^{3} \mathrm{H}\right] \mathrm{G}(1.11 \mathrm{MBq} / \mathrm{ml}$, specific activity $2.18 \mathrm{TBq} / \mathrm{mmol}$ ) in saline was infused at $0.04 \mathrm{ml} / \mathrm{min}$ during the $10-\mathrm{min}$ infusion of $3 \mu \mathrm{mol} / \mathrm{l}$
$\mathrm{MC}$, or during the 10 -min infusion of $30 \mu \mathrm{mol} / 1 \mathrm{MC}$. When present, L-NAME $(10 \mu \mathrm{mol} / \mathrm{l}$, final) was infused for $10 \mathrm{~min}$ before and throughout the graded infusions of MC. At the conclusion of these experiment, the soleus, plantaris,

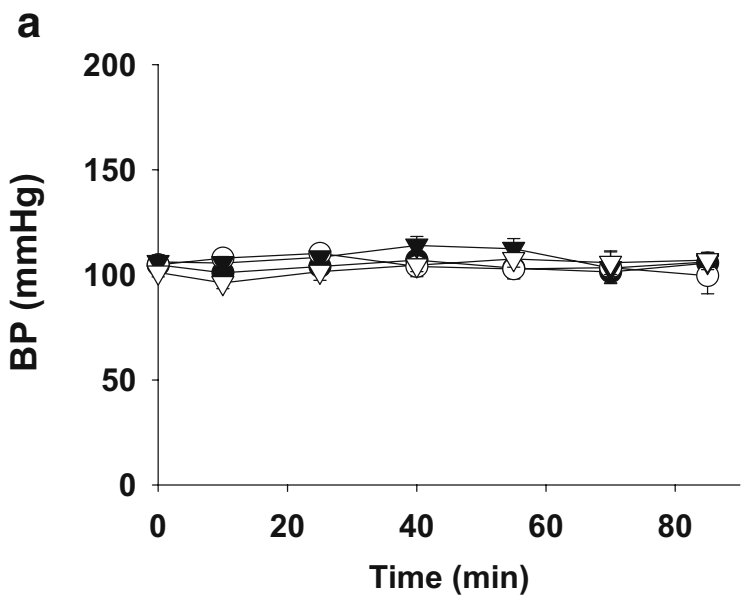

b

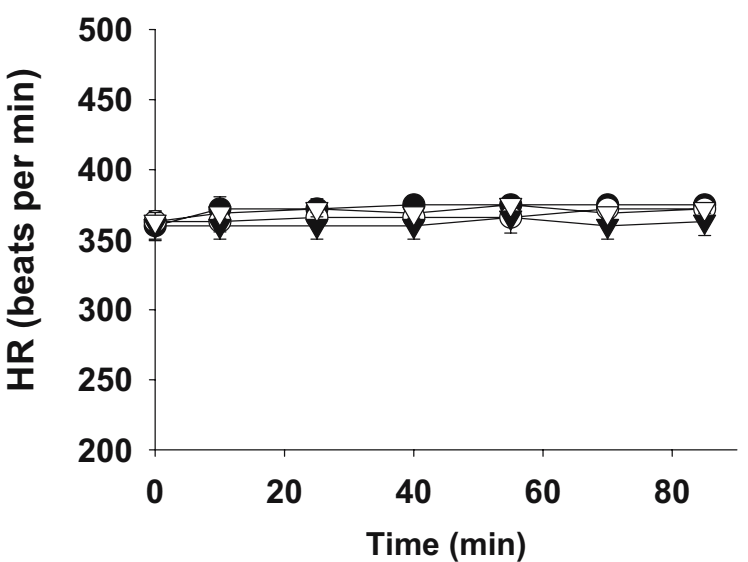

C

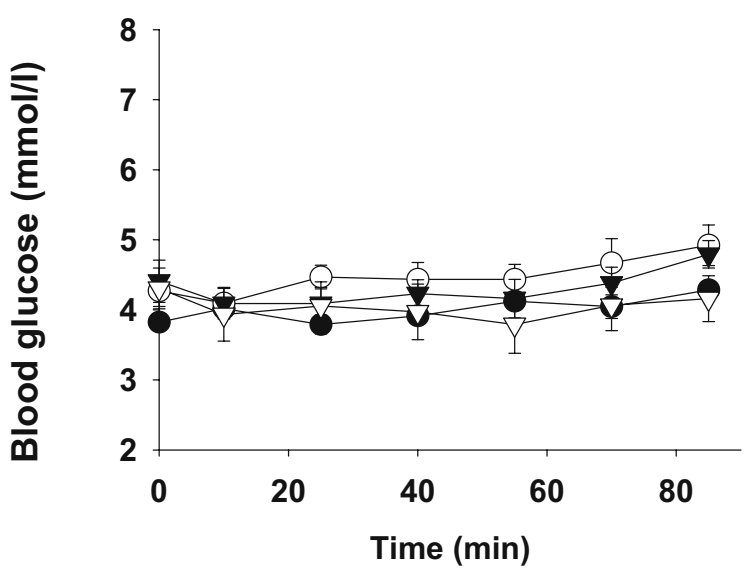

Fig. 2 Effects of MC and agents that influence NO production on a mean arterial blood pressure (BP), b heart rate (HR) and $\mathbf{c}$ blood glucose. Studies were conducted in vivo using protocol 1 (see Fig. 1) and involved infusions of L-NAME+MC (filled circles), $\mathrm{D}-\mathrm{NAME}+\mathrm{MC}$ (open circles), L-NAME+L-arginine $+\mathrm{MC}$ (filled triangles), or L-NAME alone (open triangles) into one leg only. Values are means $\pm \mathrm{SE}$ 
gastrocnemius white, gastrocnemius red, extensor digitorum longus and tibialis muscles were removed from the perfused leg, clamp frozen in liquid nitrogen and stored at $-20^{\circ} \mathrm{C}$. Individual frozen muscles were treated as described above to measure 2DG uptake.

\section{Statistical analysis}

To ascertain differences between treatment groups at the end of the experiment $(120 \mathrm{~min})$, repeated measures ANOVA was used. When a significant difference $(p<0.05)$ was found, pair-wise comparisons by the Student-Newman-Keuls test were used to determine at which individual time point differences were significant. This was used for in vivo experiments and for pressure changes in perfusion studies. For 2DG uptake (perfusion studies), one-way ANOVA was applied followed by pairwise comparisons using the Student-Newman-Keuls method. All tests were performed using the SigmaStat statistical program (Jandel Software, San Rafael, CA, USA).

\section{Results}

In vivo experiments: systemic effects

Figure 2 shows the effect of local infusion in one leg (test) of L-NAME alone, L-NAME+MC, D-NAME+MC and $\mathrm{L}-\mathrm{NAME}+\mathrm{MC}+\mathrm{L}$-arginine on systemic $\mathrm{BP}$, heart rate and blood glucose. No significant difference between any of the groups was evident, making it unlikely that either L-NAME or MC, which were infused into the test leg, spilled over into the systemic circulation.

In vivo experiments: femoral arterial blood flow

Figure 3 shows results obtained at selected intervals from continuous sampling by Transonic flow probes (Harvard Apparatus, Holliston, MA, USA) positioned around the femoral artery of each leg, test and contra-lateral control. In all experiments an initial 10-min infusion of $0.3 \mu \mathrm{mol} / \mathrm{MC}$ was made into the epigastric artery cannula of the test leg to
Fig. 3 Effects of MC and agents that influence $\mathrm{NO}$ production on FBF. Studies were conducted in vivo using protocol 1 (see Fig. 1) and involved infusions (horizontal bars) into one leg (test) only. The initial infusion of $\mathrm{MC}$ for $10 \mathrm{~min}$ at $t=0 \mathrm{~min}$ was to confirm success of the epigastric artery cannulation in the test leg. Infusions into the test leg were: D-NAME+MC (a); L-NAME + MC (b); L-NAME alone (c); and L-NAME+MC+ L-arginine (d). Values for the test (solid symbols) and contra-lateral control (open symbols) legs are shown and are means \pm SE. $* p<0.05$ for difference from contra-lateral control leg

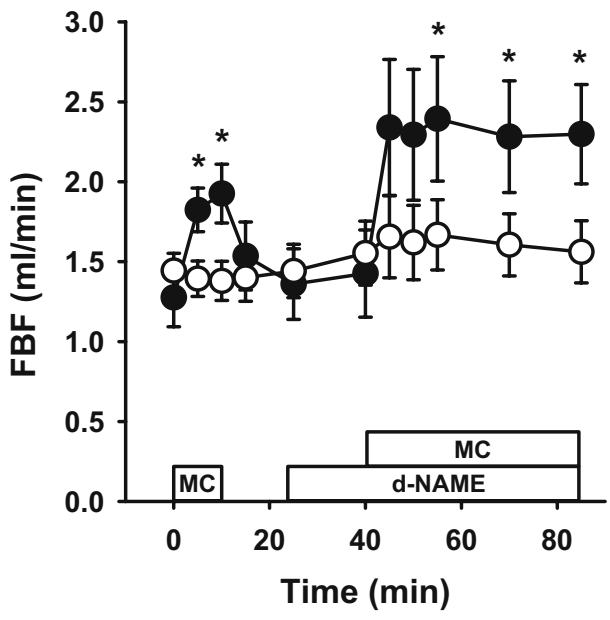

C

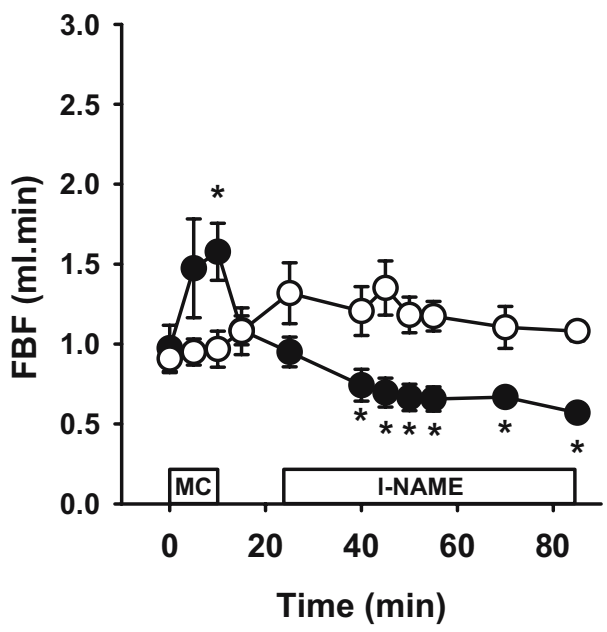

b

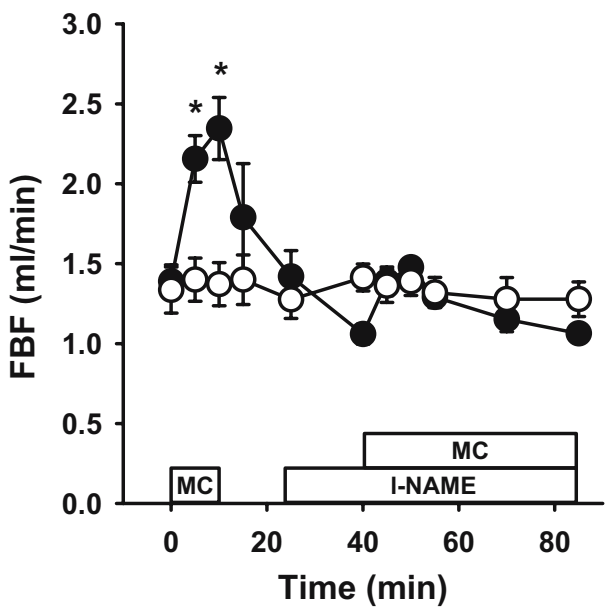

d

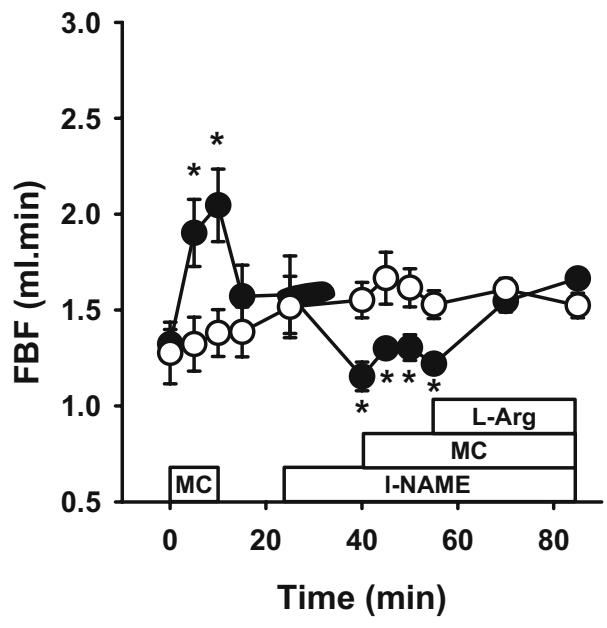


confirm successful positioning of the cannula. Following a positive response, indicated by an immediate increase in flow (Fig. 3), the FBF rate was allowed to return to basal over 15 min before experiments were commenced. At this time the NO synthase inhibitor L-NAME, or its inactive analogue D-NAME, were infused. Fifteen minutes later MC infusion was commenced and each was maintained until the end of the experiment. In Fig. 3a, $10 \mu \mathrm{mol} / 1$ D-NAME, along with $0.3 \mu \mathrm{mol} / 1 \mathrm{MC}$ were infused in the test leg. There was a significant increase in FBF of approximately $1 \mathrm{ml} / \mathrm{min}$ compared with the control leg, which remained unchanged at basal flow. When D-NAME was replaced with the active inhibitor of NO synthase, L-NAME (also $10 \mu \mathrm{mol} / \mathrm{l})$, there was no significant difference in FBF in test and control legs due to $\mathrm{MC}$ over the entire $45 \mathrm{~min}$ infusion period (Fig. 3b). However infusion of L-NAME alone (Fig. 3c) resulted in a significant reduction of FBF in the test leg by $40 \mathrm{~min}$, which remained significantly reduced for the remainder of the infusion compared with the control leg. The inclusion of $200 \mu \mathrm{mol} / \mathrm{l} \mathrm{L}$-arginine with L-NAME overcame the inhibitory influence of L-NAME, and the FBF in the test leg after L-arginine infusion was not significantly different from the control leg (Fig. 3d). Thus, these results are consistent with $\mathrm{MC}$ having both NOdependent and independent vasodilatory action.

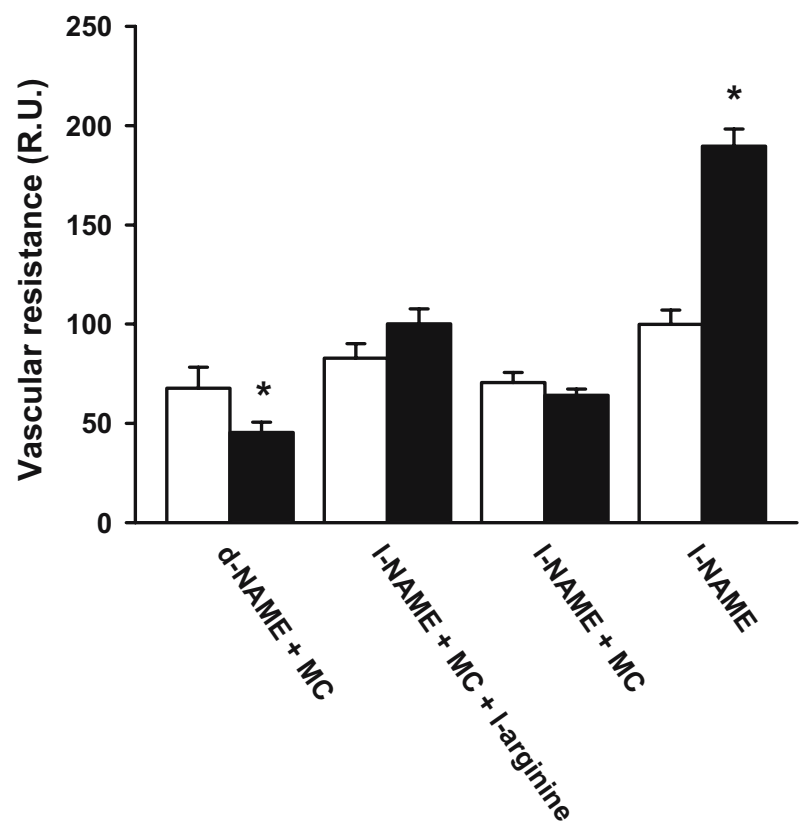

Fig. 4 Effects of MC and agents that influence NO production on vascular resistance. Studies were conducted in vivo using protocol 1 (see Fig. 1) and involved infusions into one leg (test) only. Other details were as for Fig. 3. Values (means $\pm \mathrm{SE}$ ) are expressed as resistance units $(R U)$ for the test leg (solid bars) and contra-lateral control leg (open bars) and were determined at the end of the experiment $(85 \mathrm{~min}) .{ }^{*} p<0.05$ for difference from control leg
In vivo experiments: vascular resistance

Figure 4 shows values for vascular resistance for the test and contra-lateral leg at the end of the experiment ( $85 \mathrm{~min}$ ). Vascular resistance was calculated from FBF and BP measurements at this same time point. $\mathrm{MC}$ in the presence of D-NAME resulted in a significant $(p=0.022)$ reduction in vascular resistance in the test leg compared with the control leg, but in the presence of L-NAME or L-NAME + L-arginine there was no significant difference. L-NAME infusion by itself resulted in significant $(p<0.001)$ increases in vascular resistance in the test leg compared with the control leg. The vascular resistance in the control leg for L-NAME alone infusions was significantly higher than in the controls for $\mathrm{D}-\mathrm{NAME}+\mathrm{MC}(p=0.018)$ and $\mathrm{D}-\mathrm{NAME}+\mathrm{MC}+\mathrm{L}-\operatorname{arginine}$ ( $p=0.019$ ), indicating that there may have been some spillover of L-NAME from the treated leg to the control leg. The difference was only slight (30 resistance units) compared with the effect in the test leg (120 resistance units). The vascular resistance in the test leg of L-NAME+MC treated animals was significantly higher $(p<0.001)$ than the test leg in the D-NAME+MC treated animals. This effect of L-NAME was overcome by L-arginine, as the vascular resistance in $\mathrm{L}-\mathrm{NAME}+\mathrm{MC}+\mathrm{L}$-arginine treated animals was not significantly different from D-NAME+MC.

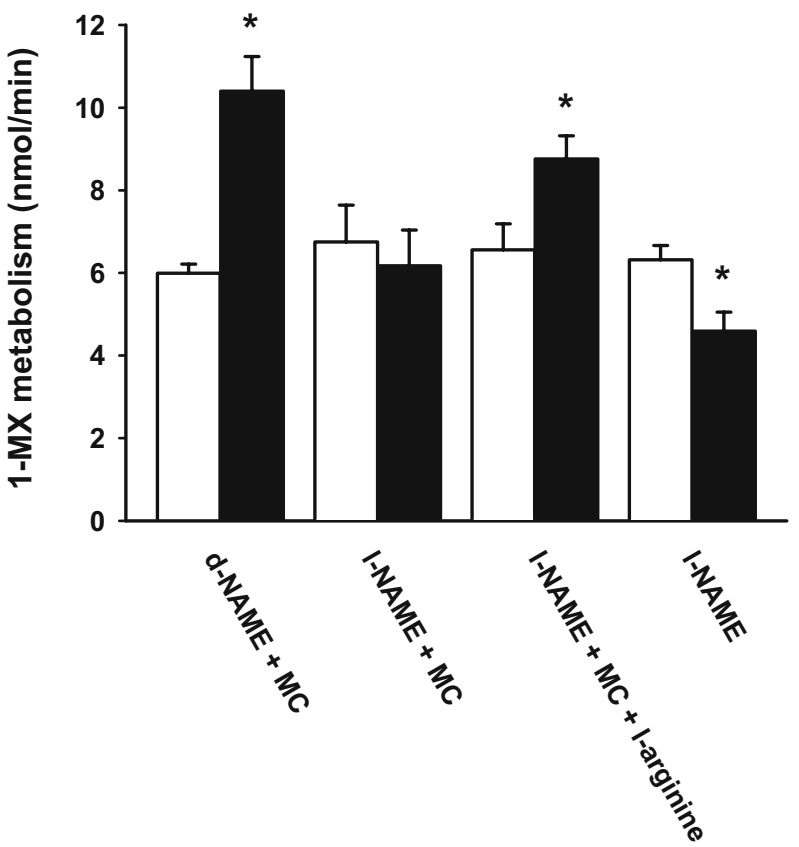

Fig. 5 Effects of MC and agents that influence NO production on 1-MX metabolism. Studies were conducted in vivo using protocol 1 (see Fig. 1) and involved infusions into one leg (test) only. 1-MX was infused systemically at $0.5 \mathrm{mg} \mathrm{min} \mathrm{mg}^{-1}$ for the last $60 \mathrm{~min}$ and arterial and femoral venous blood samples taken at the end of the experiment $(85 \mathrm{~min})$. Other details: as for Fig. 3. Values reflect 1-MX metabolism in the test (solid bars) and contra-lateral control (open bars) legs and are means \pm SE for $n=5 . * p<0.05$ for difference from corresponding control leg 
In vivo experiments: capillary recruitment

Figure 5 shows the results for 1-MX metabolism for test and contra-lateral legs where 1-MX was infused systemically for the final $60 \mathrm{~min}$ of the experiment. Local infusion of $\mathrm{MC}+$ D-NAME into the test leg significantly increased 1-MX metabolism to $10.4 \pm 0.9 \mathrm{nmol} / \mathrm{min}$ compared with the contralateral leg of $6.0 \pm 0.3 \mathrm{nmol} / \mathrm{min}(p<0.001)$. When L-NAME replaced $\mathrm{D}-\mathrm{NAME}$ the rates of $1-\mathrm{MX}$ in the test and control legs were indistinguishable. Inclusion of $\mathrm{L}$-arginine with L-NAME reversed the inhibition due to L-NAME+MC (test leg L-NAME+MC vs test leg L-NAME+MC + L-arginine, $p<0.001$; control leg vs test leg for $\mathrm{L}-\mathrm{NAME}+\mathrm{MC}+\mathrm{L}$-arginine, $p=0.002$; test leg $\mathrm{D}-\mathrm{NAME}+\mathrm{MC}$ vs test leg L-NAME $+\mathrm{MC}+$ $\mathrm{L}$-arginine, $\mathrm{NS}, p=0.079)$. Infusion of L-NAME alone into the test leg reduced the metabolism of 1-MX to lower than that of the control leg $(p=0.01)$. These results are similar to the effects of L-NAME on MC-mediated changes in FBF and vascular resistance, again suggesting that the vascular actions of $\mathrm{MC}$ have both NO-dependent and -independent components.

In vivo experiments: insulin

Plasma insulin values before $(-5 \mathrm{~min})$ and after $\mathrm{MC}$ (85 $\mathrm{min})$ with the treatment as shown in parentheses were $285 \pm 151$ and $263 \pm 147 \mathrm{pmol} / 1$ (D-NAME), 460 \pm 161 and $499 \pm 218 \mathrm{pmol} / 1$ (L-NAME), and $238 \pm 37$ and $309 \pm$ $21 \mathrm{pmol} / 1$ (L-NAME+arginine) respectively. No significant effect of MC was seen, either with or without D-NAME, L-NAME or L-NAME+arginine, on the plasma insulin

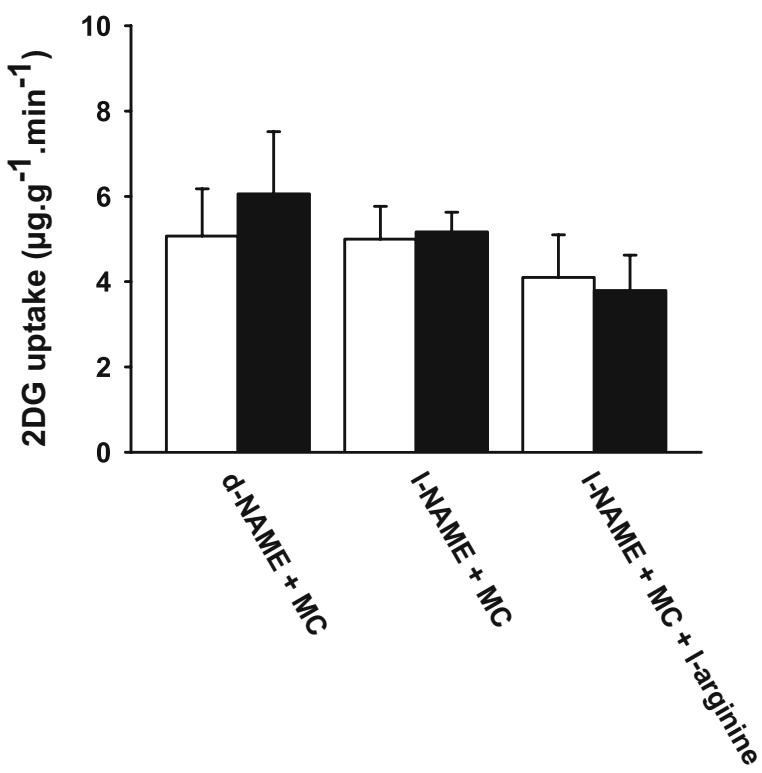

Fig. 6 Effects of MC and agents that influence NO production on 2DG uptake. Studies were conducted in vivo using protocol 1 (see Fig. 1) and involved infusions into one leg (test) only. Radioactive 2DG was injected systemically as a bolus at $40 \mathrm{~min}$ and muscles were sampled at the end of the experiment $(85 \mathrm{~min})$. Other details: as for Fig. 3. Values for combined lower leg muscles of the test (solid bars) and contra-lateral control (open bars) legs are shown and are means \pm SE levels. Similarly, there was no significant difference between the three experimental groups and thus plasma insulin levels were unlikely to have affected muscle glucose uptake or capillary recruitment.

In vivo experiments: glucose uptake

Figure 6 shows glucose uptake measured by 2DG uptake of the combined muscles (soleus, plantaris, gastrocnemius,

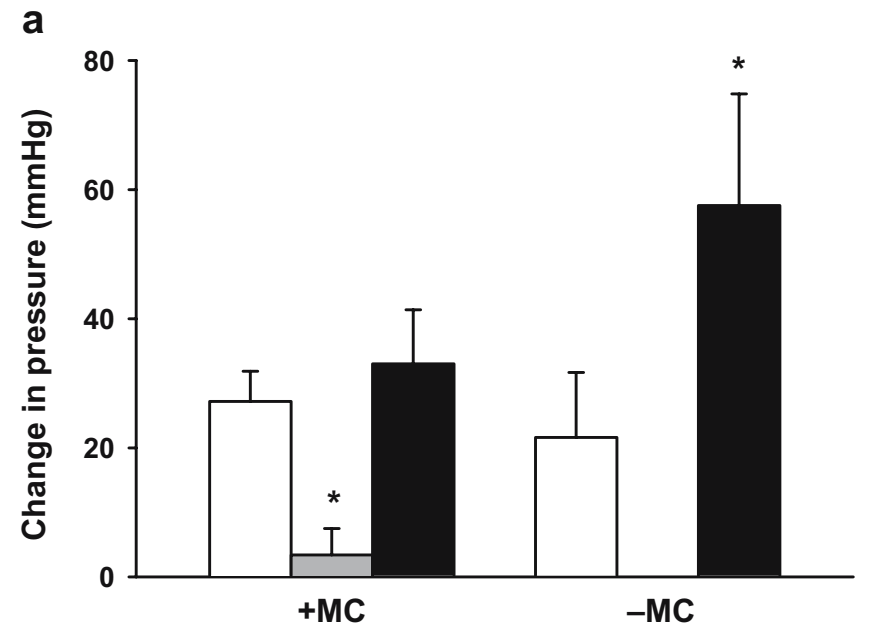

b

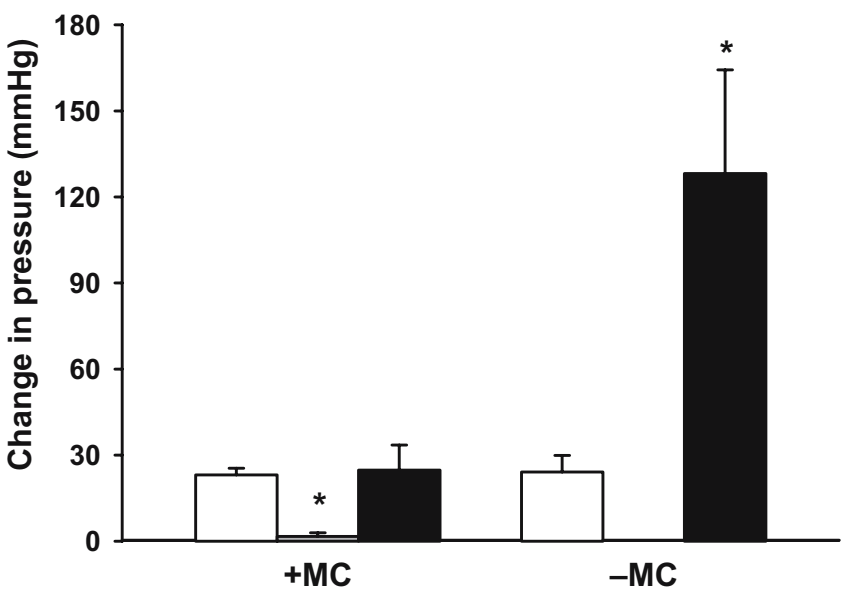

Fig. 7 Vasodilatory effects of MC on pre-constricted hindlimb. Studies were conducted using the constant-flow pump-perfused rat hindlimb as shown by protocol 1 (see Fig. 1). The hindlimb was preconstricted by constant infusion of $70 \mathrm{nmol} / 1 \mathrm{NA}$ (a) or $300 \mathrm{nmol} / \mathrm{l}$ 5 -HT (b). Infusion of $0.6 \mu \mathrm{mol} / 1 \mathrm{MC}$ began $10 \mathrm{~min}$ after commencement of either NA or 5-HT. In those experiments receiving L-NAME infusion, this was commenced 5 min after MC. Perfusion pressure values for NA or 5-HT alone (white bars) were taken immediately preceding $\mathrm{MC}$ infusion; values for $\mathrm{NA}+\mathrm{MC}$ or 5-HT+MC (grey bars) were taken immediately preceding $\mathrm{L}-\mathrm{NAME}$ infusion, and values for $\mathrm{NA}+\mathrm{MC}+\mathrm{L}-\mathrm{NAME}$ (black bars) were taken at $30 \mathrm{~min}$, corresponding to the end of the experiment, as indicated in protocol 2 of Fig. 1. In separate experiments MC was replaced by saline $(-\mathrm{MC})$. NA or 5-HT were infused alone for $15 \mathrm{~min}$ prior to the infusion of L-NAME; pressure readings were taken at the same times as when MC was present. All values shown are means $\pm \mathrm{SE}(n=6) . * p<0.05$ for difference from all other groups (one-way repeated measures ANOVA) 
extensor digitorum longus and tibialis) from the lower leg. Although there was a trend for MC+D-NAME to increase 2DG uptake in the test leg, this was not significant. In addition, no significant differences between test and control leg were seen for the combinations MC+L-NAME, or $\mathrm{MC}+\mathrm{L}-\mathrm{NAME}+\mathrm{L}-$ arginine. Only results for the combined lower leg muscle are shown; results from individual muscles did not reveal any significant differences between any of the three combinations, $\mathrm{MC}+\mathrm{D}-\mathrm{NAME}, \mathrm{MC}+$ $\mathrm{L}-\mathrm{NAME}$, or $\mathrm{MC}+\mathrm{L}-\mathrm{NAME}+\mathrm{L}-$ arginine (results not shown).

Perfused hindlimb: vasodilatory effects of MC

Figure 7 shows the effect of $10 \mu \mathrm{mol} / \mathrm{l}$ L-NAME, with and without $0.6 \mu \mathrm{mol} / 1 \mathrm{MC}$, on perfusion pressure of the constant-flow pump-perfused rat hindlimb pre-constricted with either $70 \mu \mathrm{mol} / 1 \mathrm{NA}$ or $300 \mu \mathrm{mol} / 15$-HT. MC alone significantly dilated the constriction simulated by NA $(p<0.05)$ and the dilatation was reversed by $10 \mu \mathrm{mol} / 1$ L-NAME (Fig. 7a). However, since the combination of NA+L-NAME significantly increased the vasoconstriction by NA, it was apparent that MC had vasodilatory action that was not totally blocked by L-NAME. Similar results were observed for the vasoconstriction due to 5-HT, as MC significantly dilated the 5-HT-mediated vasoconstriction and this dilatation was blocked by L-NAME (Fig. 7b). However, L-NAME when added to 5-HT alone potentiated the vasoconstriction due to $5-\mathrm{HT}$.

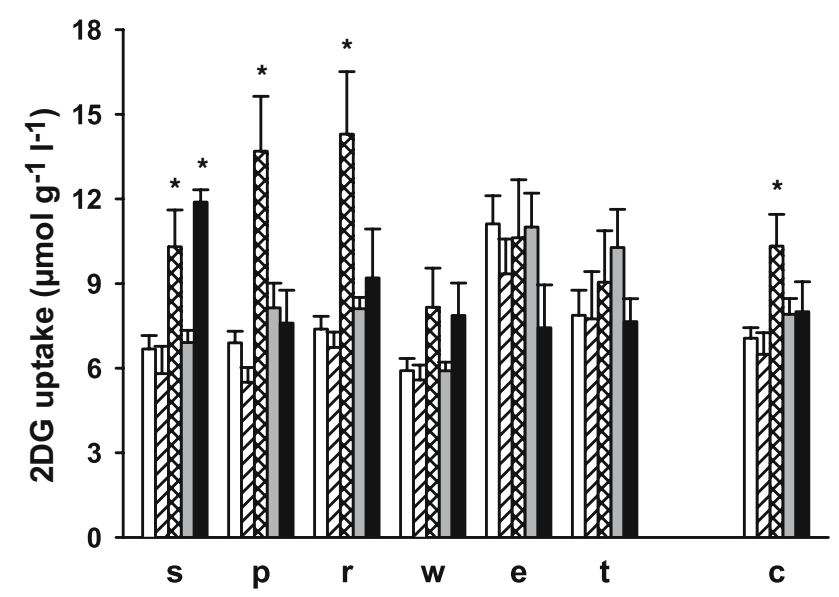

Fig. 8 Effect of $\mathrm{MC}$ on hindlimb 2DG uptake. Studies were conducted using the constant-flow pump-perfused rat hindlimb. Infusions of increasing doses of $0.3,3,15$ and $30 \mu \mathrm{mol} / 1 \mathrm{MC}$ were made at step-wise intervals of $10 \mathrm{~min}$. Radioactive $2 \mathrm{DG}$ was infused for the entire $10 \mathrm{~min}$ period during either 3 or $30 \mu \mathrm{mol} / \mathrm{l} \mathrm{MC}$. L-NAME $(10 \mu \mathrm{mol} / \mathrm{l})$ infusion was started 20 and $40 \mathrm{~min}$ prior to infusion of 3 and $30 \mu \mathrm{mol} / 1 \mathrm{MC}$, respectively. Muscles were sampled for the determination of 2DG uptake at the end of the perfusion. $s$ soleus, $p$ plantaris, $r$ red gastrocnemius, $w$ white gastrocnemius, $e$ extensor digitorum longus, $t$ tibialis, $c$ combined. Open bars, saline $(154 \mathrm{mmol} / 1 \mathrm{NaCl})$; hatched bars, $3 \mu \mathrm{mol} / 1 \mathrm{MC}$; double-hatched bars, $30 \mu \mathrm{mol} / 1 \mathrm{MC}$; grey bars, $30 \mu \mathrm{mol} / 1 \mathrm{MC}+$ L-NAME; black bars, L-NAME alone. * $p<0.05$ for difference from saline (one-way ANOVA on each muscle)
Perfused hindlimb: effects of $\mathrm{MC}$ on glucose uptake

Figure 8 shows data for $2 \mathrm{DG}$ uptake in muscles of the lower leg for saline $(154 \mathrm{mmol} / \mathrm{l} \mathrm{NaCl}), 3$ or $30 \mu \mathrm{mol} / \mathrm{l} \mathrm{MC}$, $30 \mu \mathrm{mol} / 1 \mathrm{MC}+10 \mu \mathrm{mol} / 1 \mathrm{~L}-\mathrm{NAME}$ and L-NAME alone. 2DG uptake was significantly increased for soleus, plantaris and red gastrocnemius, as well as the combined muscles, by $30 \mu \mathrm{mol} / 1 \mathrm{MC}$, and this was blocked by L-NAME. L-NAME alone significantly increased 2DG uptake in the soleus muscle but not in the other muscles, nor did it influence the outcome for the combined group.

\section{Discussion}

Findings from the present study support our hypothesis that vascular responses to $\mathrm{MC}$ occur at lower doses than the direct myocyte MC-mediated increase in muscle glucose uptake. Thus, in the constant-flow pump-perfused rat hindlimb, MC stimulated glucose uptake at $30 \mu \mathrm{mol} / 1$, but was without effect at $3 \mu \mathrm{mol} / \mathrm{l}$, even though it was able to fully vasodilate pre-constriction by either NA or 5-HT at onefifth of that dose. The implication of these findings is that MC may influence glucose metabolism in vivo by two discrete effects that are concentration dependent. At low doses the vascular effect of vasodilatation is likely to have beneficial effects on nutrient and hormone delivery by enhancing microvascular perfusion (capillary recruitment), as has been shown by us previously [13]. At the higher dose of $\mathrm{MC}(30 \mu \mathrm{mol} / 1)$ the two effects would very likely occur concomitantly, with enhanced delivery of nutrients, hormones and $\mathrm{MC}$ itself to enhance myocyte glucose uptake. However, since MC itself is not a naturally occurring agonist, but rather a congener of acetylcholine, it is difficult to know if levels of $30 \mu \mathrm{mol} / 1 \mathrm{MC}$ simulate physiologically meaningful levels of acetylcholine. Most studies employing acetylcholine use $\leq 1 \mu \mathrm{mol} / 1$. In any event, we show that the direct myocyte effect of $\mathrm{MC}$ at $30 \mu \mathrm{mol} / 1$ to increase glucose uptake is fully NO dependent, but the vascular effects at the lower doses are only partially NO dependent.

The present findings represent a follow-up of a previous study from this laboratory where locally administered MC $(0.3 \mu \mathrm{mol} / \mathrm{l})$ into one leg of a rat in vivo was found to enhance capillary recruitment and glucose uptake by systemic physiological hyperinsulinaemia [13]. Thus it would be reasonable to conclude that $\mathrm{MC}$ has enhanced insulin action largely by its vascular actions as the concentration used in that study [13] was well below that presently shown to have direct stimulatory effects on muscle glucose uptake. In the present study, low dose of MC ${ }^{D}-N A M E$ tended to, but did not significantly increase glucose uptake by muscle, which might suggest that the positive effect on delivery seen by an increase in capillary recruitment by this combination was unable to potentiate basal insulin effects. This finding was similar to that previously observed with MC alone [13], where the tendency to increase glucose uptake was also not significant. However, when the data from the two studies are combined on the assumption that $\mathrm{D}-\mathrm{NAME}+\mathrm{MC}$ is effectively equivalent to $\mathrm{MC}$ alone, the 
difference becomes significant $(p<0.05)$. Thus, the large increase in capillary recruitment due to $\mathrm{MC}$ is able to potentiate basal insulin-mediated glucose uptake, even though the effect is small. A relatively small effect of MC to potentiate basal insulin action may be because the concentration of insulin required to increase muscle glucose uptake in the rat is at least twice basal levels [19].

It is still largely controversial whether glucose and insulin delivery to muscle has an impact on overall glucose uptake. It is known that the interstitial fluid concentration of glucose is considerably lower than plasma glucose [20], probably as a result of capillary diffusion capacity of glucose being a rate-limiting step for glucose metabolism. When insulin resistance is present, a combination of diminished myocellular glucose uptake and delivery may occur [20], and there have been reports that interstitial glucose concentrations may either increase [21], or decrease [20], depending on whether the defect in myocyte glucose transport is greater or less than the defect in glucose delivery. The question of glucose delivery as a rate-limiting step for muscle glucose uptake has been partly addressed by considering the relationship between bulk blood flow and glucose uptake. For example, Grubb and Snarr [22], using the perfused rat hindlimb system, showed that increasing glucose concentration within the physiological range increased glucose uptake in a linear fashion. However, with a constant glucose level, the relationship between flow and glucose uptake was hyperbolic. They proposed that the plateau effect of glucose uptake was due to flow being shunted into a non-nutritive route. However, it was clear that at physiological flow rates, glucose uptake was not maximal and flow-mediated uptake occurred. Just why flow-mediated glucose uptake occurs requires a consideration of glucose extraction. For a simplified model of a single capillary of fixed surface area, glucose uptake is limited either by permeability or flow. Assuming complete permeability of the capillary to glucose, then depending on metabolism the extraction will be $100 \%$ and thus flow could become rate limiting. However, in a situation in which the extraction is low, an increase in flow will have no effect on the rate of glucose uptake [12]. Since in vivo approximately $40 \%$ of glucose is extracted in insulinstimulated muscle [23], the situation is a combination of the two scenarios. A concentration gradient forms from the arterial to the venous end of the capillary as glucose is extracted. This gradient is reduced by increasing flow, although it was estimated that in vivo, this would only increase glucose uptake by approximately $10 \%$ [23]. If capillary recruitment also takes place, the capillary surface area will be increased, leading to further enhancement of glucose uptake. Natali [24] has compared experimental data with a model of gradient dilution and capillary recruitment. In the capillary recruitment model, glucose uptake increases in a linear fashion in proportion to flow. In contrast, in the gradient dilution scenario, an increase in flow was predicted to cause a hyperbolic increase in glucose uptake. In the actual experiments [24], flow was altered during insulin infusion. It was found that the data fitted a model of capillary recruitment when flow was reduced by ouabain and a model of gradient dilution after increasing flow with phentolamine and propranolol. Such data suggested that increased flow on its own would have little effect on glucose uptake.

One way to manipulate bulk flow to muscle has been to use vasodilators, and since there has been sufficient reason to believe that flow could be a limiting factor particularly in insulin-resistant muscle, vasodilators have been acutely administered in an attempt to address this issue. However, based on the arguments in the previous paragraph, vasodilators that increase total limb blood flow, such as adenosine [6], bradykinin [7], sodium nitroprusside [8], low doses of IGF-1 [9] and adrenaline [10], would not be expected to increase glucose uptake or overcome insulin resistance. However, not all vasodilators may act in the same way, and MC may be one such example. For example, Baron et al. [25] reported MC to further increase flow and the NO synthase inhibitor L-NMMA to decrease flow during a hyperinsulinaemiceuglycaemic clamp. The changes in limb blood flow were paralleled by changes in glucose uptake in the same limb. Thus, glucose uptake was increased by $\mathrm{MC}$ and the increase was blocked by L-NMMA. Because the data indicated a significant deviation from the Renkin equation for a fixed capillary surface area [12], the authors concluded that capillary recruitment was responsible [25]. Similar data were reported by Sarabi et al. [3], who found that MC, but not nitroprusside, increased both forearm blood flow and glucose uptake in hypertensive patients. Recently, we have reported that MC, but not bradykinin, potentiated insulin-mediated capillary recruitment and glucose uptake in rat muscle in vivo [13]. However, it was not clear that the metabolic effects of MC could be entirely attributed to the vascular action, particularly since NO donors can increase muscle glucose uptake independently of vascular effects $[4,5]$. The present findings strongly support the suggestion that the enhancement by MC is attributable to a vascular effect.

Reports linking glucose uptake by muscle to the direct effects of NO have largely originated from experiments where isolated muscles have been incubated with NO donors [4,5], agents known to increase intracellular cyclic GMP [4], or both [4]. Support for NO involvement in muscle glucose uptake in vivo, where vascular effects to alter nutrient and hormone delivery cannot be entirely ruled out, is fragmentary. There are two major effectors of muscle glucose uptake, insulin and exercise, and both have been claimed to involve some dependency on NO [1-3, 26]. In rats, insulin-mediated increase in limb blood flow is blocked by systemically administered NO synthase inhibitors [27, 28], as is insulin-mediated capillary recruitment $[27,28]$ and glucose uptake $[27,28]$, although the latter is controversial in humans $[24,29,30]$. However, since a centrally injected NO synthase inhibitor can mimic the insulin resistance and hypertension seen with systemic NO synthase inhibitors [31], a local mechanism involving endothelial insulin receptors, endothelial NO synthase and NO-dependent vasodilatation is far from clear. In addition, the vascular endothelial insulin receptor knock-out mouse is not insulin resistant [32], unless subjected to low-salt diet. There are arguments for [26] and against [33] NO 
involvement in exercise-mediated blood flow or oxygen uptake. In one study an NO synthase inhibitor used during exercise in vivo inhibited glucose uptake without change in flow [34], suggesting a direct metabolic effect.

The findings from the present study show that the locally infused low-dose vasodilatory action of $\mathrm{MC}$ in vivo is not completely NO-dependent and thus raises questions regarding the cholinergic receptors involved. In human forearm, characterisation of vasodilating muscarinic receptors suggests that cholinergic vasodilation in this vascular bed is predominantly mediated by the M3-receptor subtype [35, 36]. In rodents the receptor subtype in vasculature of the skeletal muscle is yet to be identified, and so far a major role of M3-cholinergic vasodilatation in the perfused mesenteric vascular bed has been described in both the spontaneously hypertensive rat and the corresponding control strain (WistarKyoto) [37]. These receptors are able to vasodilate using NOdependent and NO-independent endothelial hyperpolarising factor-mediated mechanisms [38]. However, although mice lacking M3 subtype receptors have been generated [39], it has not been reported that these animals have defects relevant to glucose homeostasis, even though they lack smooth muscle contraction response to cholinergic agonists [40].

In summary, a dose-dependent dichotomy of MC was observed. At low dose $(0.3-3 \mu \mathrm{mol} / \mathrm{l}), \mathrm{MC}$ is a potent vasodilator in muscle in vivo and in vitro without metabolic effects, but at higher doses $(\geq 30 \mu \mathrm{mol} / \mathrm{l})$ there is a direct metabolic effect of MC leading to increased glucose uptake. Both the vascular and metabolic effects are sensitive to L-NAME, but the metabolic effects are fully NO-dependent, while the vascular effects are only partially NO-dependent.

Acknowledgements This work was supported in part by grants from the National Health and Medical Research Council, and the Heart Foundation of Australia. S. Rattigan is a Heart Foundation Career Fellow and J. M. B. Newman is an Australian Research Council Fellow.

\section{References}

1. Anderson TJ, Meredith IT, Ganz P, Selwyn AP, Yeung AC (1994) Nitric oxide and nitrovasodilators: similarities, differences and potential interactions. J Am Coll Cardiol 24:555-566

2. Steinberg HO, Brechtel G, Johnson A, Fineberg N, Baron AD (1994) Insulin-mediated skeletal muscle vasodilation is nitric oxide dependent. A novel action of insulin to increase nitric oxide release. J Clin Invest 94:1172-1179

3. Sarabi M, Lind L, Millgard J et al (1999) Local vasodilatation with metacholine, but not with nitroprusside, increases forearm glucose uptake. Physiol Res 48:291-295

4. Etgen GJJ, Fryburg DA, Gibbs EM (1997) Nitric oxide stimulates skeletal muscle glucose transport through a calcium/contraction- and phosphatidylinositol-3-kinase-independent pathway. Diabetes 46:1915-1919

5. Young ME, Radda GK, Leighton B (1997) Nitric oxide stimulates glucose transport and metabolism in rat skeletal muscle in vitro. Biochem J 322:223-228

6. Natali A, Bonadonna R, Santoro D et al (1994) Insulin resistance and vasodilation in essential hypertension. Studies with adenosine. J Clin Invest 94:1570-1576
7. Nuutila P, Raitakari M, Laine H et al (1996) Role of blood flow in regulating insulin-stimulated glucose uptake in humans. Studies using bradykinin, $\left[{ }^{15} \mathrm{O}\right]$ water, and $\left[{ }^{18} \mathrm{~F}\right]$ fluoro-deoxyglucose and positron emission tomography. J Clin Invest 97:1741-1747

8. Natali A, Quinones GA, Pecori N, Sanna G, Toschi E, Ferrannini E (1998) Vasodilation with sodium nitroprusside does not improve insulin action in essential hypertension. Hypertension 31:632-636

9. Pendergrass M, Fazioni E, Collins D, Defronzo RA (1998) IGF-I increases forearm blood flow without increasing forearm glucose uptake. Am J Physiol 275:E345-E350

10. Rattigan S, Clark MG, Barrett EJ (1997) Hemodynamic actions of insulin in rat skeletal muscle: evidence for capillary recruitment. Diabetes 46:1381-1388

11. Baron AD, Tarshoby M, Hook G et al (2000) Interaction between insulin sensitivity and muscle perfusion on glucose uptake in human skeletal muscle: evidence for capillary recruitment. Diabetes 49:768-774

12. Renkin EM (1984) Control of microcirculation and bloodtissue exchange. In: Renkin EM, Michel CC, Geiger SR (eds) Handbook of physiology - the cardiovascular system IV. American Physiological Society, Bethesda, pp 627-687

13. Mahajan H, Richards SM, Rattigan S, Clark MG (2004) Local methacholine but not bradykinin potentiates insulin-mediated glucose uptake in muscle in vivo by augmenting capillary recruitment. Diabetologia 47:2226-2234

14. Kraegen EW, James DE, Jenkins AB, Chisholm DJ (1985) Dose-response curves for in vivo insulin sensitivity in individual tissues in rats. Am J Physiol 248:E353-E362

15. James DE, Jenkins AB, Kraegen EW (1985) Heterogeneity of insulin action in individual muscles in vivo: euglycemic clamp studies in rats. Am J Physiol 248:E567-E574

16. Ruderman NB, Houghton CR, Hems R (1971) Evaluation of the isolated perfused rat hindquarter for the study of muscle metabolism. Biochem J 124:639-651

17. Colquhoun EQ, Hettiarachchi M, Ye JM et al (1988) Vasopressin and angiotensin II stimulate oxygen uptake in the perfused rat hindlimb. Life Sci 43:1747-1754

18. Clark MG, Colquhoun EQ, Rattigan S et al (1995) Vascular and endocrine control of muscle metabolism. Am J Physiol 268: E797-E812

19. Zhang L, Vincent MA, Richards SM et al (2004) Insulin sensitivity of muscle capillary recruitment in vivo. Diabetes 53:447-453

20. Halseth AE, Bracy DP, Wasserman DH (2000) Limitations to basal and insulin-stimulated skeletal muscle glucose uptake in the high-fat-fed rat. Am J Physiol 279:E1064-E1071

21. Holmang A, Muller M, Andersson OK, Lonnroth P (1998) Minimal influence of blood flow on interstitial glucose and lactate-normal and insulin-resistant muscle. Am J Physiol 274: E446-E452

22. Grubb B, Snarr JF (1977) Effect of flow rate and glucose concentration on glucose uptake rate by the rat limb. Proc Soc Exp Biol Med 154:33-36

23. Baron AD (1994) Hemodynamic actions of insulin. Am J Physiol 267:E187-E202

24. Natali A (1997) Skeletal muscle blood flow and insulin action. Nutr Metab Cardiovasc Dis 7:105-109

25. Baron AD, Tarshoby M, Hook G et al (2000) Interaction between insulin sensitivity and muscle perfusion on glucose uptake in human skeletal muscle: evidence for capillary recruitment. Diabetes 49:768-774

26. Chavoshan B, Sander M, Sybert TE, Hansen J, Victor RG, Thomas GD (2002) Nitric oxide-dependent modulation of sympathetic neural control of oxygenation in exercising human skeletal muscle. J Physiol 540:377-386

27. Vincent MA, Barrett EJ, Lindner JR, Clark MG, Rattigan S (2003) Inhibiting NOS blocks microvascular recruitment and blunts glucose uptake in response to insulin. Am J Physiol 285: E123-E129 
28. Vincent MA, Clerk LH, Lindner JR et al (2004) Microvascular recruitment is an early insulin effect that regulates skeletal muscle glucose uptake in vivo. Diabetes 53:1418-1423

29. Baron AD, Steinberg HO, Chaker H, Leaming R, Johnson A, Brechtel G (1995) Insulin-mediated skeletal muscle vasodilation contributes to both insulin sensitivity and responsiveness in lean humans. J Clin Invest 96:786-792

30. Scherrer U, Randin D, Vollenweider P, Vollenweider L, Nicod $P$ (1994) Nitric oxide release accounts for insulin's vascular effects in humans. J Clin Invest 94:2511-2515

31. Shankar R, Zhu JS, Ladd B, Henry D, Shen HQ, Baron AD (1998) Central nervous system nitric oxide synthase activity regulates insulin secretion and insulin action. J Clin Invest 102:1403-1412

32. Vicent D, Ilany J, Kondo T et al (2003) The role of endothelial insulin signaling in the regulation of vascular tone and insulin resistance. J Clin Invest 111:1373-1380

33. Frandsen U, Bangsbo J, Sander M et al (2001) Exerciseinduced hyperaemia and leg oxygen uptake are not altered during effective inhibition of nitric oxide synthase with $N(\mathrm{G})$ nitro-L-arginine methyl ester in humans. J Physiol 531:257-264

34. Bradley SJ, Kingwell BA, McConell GK (1999) Nitric oxide synthase inhibition reduces leg glucose uptake but not blood flow during dynamic exercise in humans. Diabetes 48:1815-1821
35. Bruning TA, Hendriks MG, Chang PC, Kuypers EA, van Zwieten PA (1994) In vivo characterization of vasodilating muscarinic-receptor subtypes in humans. Circ Res 74:912-919

36. Bruning TA, Chang PC, Hendriks MG, Vermeij P, Pfaffendorf M, van Zwieten PA (1995) In vivo characterization of muscarinic receptor subtypes that mediate vasodilatation in patients with essential hypertension. Hypertension 26:70-77

37. Hendriks MG, Pfaffendorf M, van Zwieten PA (1993) Characterization of the muscarinic receptors in the mesenteric vascular bed of spontaneously hypertensive rats. J Hypertens $11: 1329-1335$

38. McCulloch AI, Bottrill FE, Randall MD, Hiley CR (1997) Characterization and modulation of EDHF-mediated relaxations in the rat isolated superior mesenteric arterial bed. $\mathrm{Br} \mathrm{J}$ Pharmacol 120:1431-1438

39. Matsui M, Motomura D, Karasawa $\mathrm{H}$ et al (2000) Multiple functional defects in peripheral autonomic organs in mice lacking muscarinic acetylcholine receptor gene for the M3 subtype. Proc Natl Acad Sci U S A 97:9579-9584

40. Matsui M, Motomura D, Fujikawa T et al (2002) Mice lacking M2 and M3 muscarinic acetylcholine receptors are devoid of cholinergic smooth muscle contractions but still viable. J Neurosci 22:10627-10632 